\title{
SCIENTIFIC REPORTS

\section{OPEN Maternal high fat diet consumption reduces liver alpha7 nicotinic cholinergic receptor expression and impairs insulin signalling in the offspring}

S. O. Costa ${ }^{1}$, C. M. Souza ${ }^{1}$, P. G. Lanza ${ }^{1}$, J. O. Sartori ${ }^{1}$, L. M. Ignacio-Souza ${ }^{1,3}$, T. Candreva $^{2}$, H. G. Rodrigues $\mathbb{D}^{2}$, A. S. Torsoni ${ }^{1,3}{ }^{3}$ M. Milanski ${ }^{1,3}$ \& M. A. Torsoni $i^{1,3^{*}}$

The activation of nicotinic acetylcholine receptor $\alpha 7$ subunit $(\alpha 7 n A C h R)$ has been associated to anti-inflammatory response in macrophages. High-fat diet (HFD) consumption during pregnancy and lactation impairs the cholinergic anti-inflammatory pathway in liver and white adipose tissue of offspring. In order to evaluate the relationship between damage in the cholinergic anti-inflammatory pathway and insulin resistance (IR) development, the liver of offspring of obese dams was investigated. Additionally, the capacity of $\alpha 7 \mathrm{nAChR}$ activation to reduce IR induced by saturated fatty acid was investigated in hepatoma cell line. Initially, female mice were subjected to either standard chow (SC) or HFD during pregnancy and lactation period. After weaning, only male offspring from HFD dams (HFDO) and SC dams (SC-O) were fed with the SC diet. Hepatic $\alpha 7 n A C h R$ expression was downregulated, and hepatic TNF- $\alpha$, IL-1 $\beta$, and pIKK level, but not $\mathrm{PJNK}$, were elevated in the HFD-O compared to SC-O mice. Besides, hepatic expression of TNF- $\alpha$ in response to lipopolysaccharide (LPS) was higher in HFD-O than SC-O mice. Insulin-stimulated phosphorylation of the AKT was lower in HFD-O compared to SC-O. Additionally, insulin-stimulated phosphorylation of the AKT in KO $\alpha 7^{A l b-C r e ~}$ mice fed HFD was lower than WT mice fed HFD. In hepatoma cell line, palmitate increased IL- 6 and TNF- $\alpha$ expressions and $\mathrm{pJNK}$ level. These effects were accompanied by reduced capacity of insulin to stimulate AKT phosphorylation. PNU or nicotine reduced cytokine expression and JNK activation, but improved insulin resistance induced by palmitate. Our results suggest that maternal obesity impairs hepatic $\alpha 7 n A C h R$ expression and AKT phosphorylation in the offspring. In vitro studies suggest that $\alpha 7 \mathrm{nAChR}$ activation has potential to reduce deleterious effect of saturated fatty acids on insulin signalling.

Cholinergic anti-inflammatory pathway activation reduces inflammatory cytokines expression through the activation of nicotinic acetylcholine receptor $\alpha 7$ subunit $(\alpha 7 \mathrm{nAChR})$ by acetylcholine ${ }^{1,2}$. This mechanism was first described to occur in macrophages, and it comprises an afferent arm that senses inflammation and an efferent arm that inhibits innate immune responses ${ }^{1,3}$. Activation of anti-inflammatory cholinergic pathway by agonist nicotine inhibits the inflammatory response, while antagonist of $\alpha 7 \mathrm{nAChR}$ blocks the anti-inflammatory effect $^{4,5}$. Moreover, mice $\alpha 7 \mathrm{nAChR}^{-1-}$ produces significantly more LPS-induced TNF- $\alpha$, IL- $1 \beta$ and other cytokines systemically, and the electrical stimulation of the vagus nerve was ineffective to attenuate LPS-induced inflammation ${ }^{6}$.

Macrophage activation and inflammation are elevated in obesity. Characteristics of obesity-induced inflammation include elevated production of proinflammatory molecules by adipose tissue and activation of a network of inflammatory signalling pathways. These are important factors for the development of insulin resistance $e^{7,8}$.

\footnotetext{
${ }^{1}$ Laboratory of Metabolic Disorders, School of Applied Sciences, University of Campinas, Campinas, Brazil. ${ }^{2}$ Laboratory of Nutrients and Tissue Repair, School of Applied Sciences, University of Campinas, Campinas, Brazil. ${ }^{3}$ Obesity and Comorbidities Research Center, University of Campinas, Campinas, Brazil. *email: marcio.torsoni@ fca.unicamp.br
} 
a

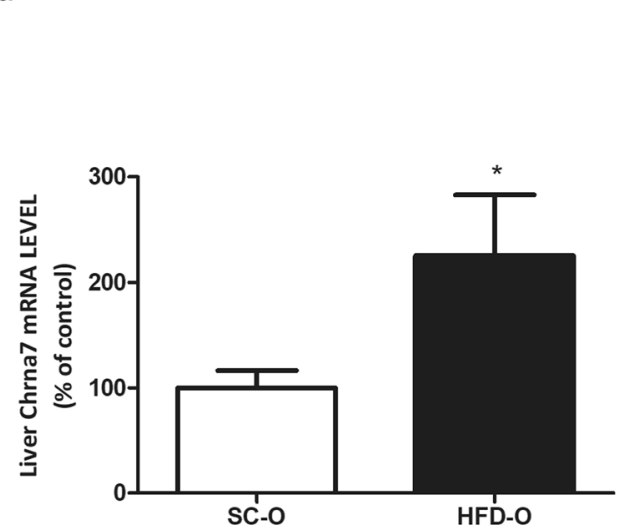

b
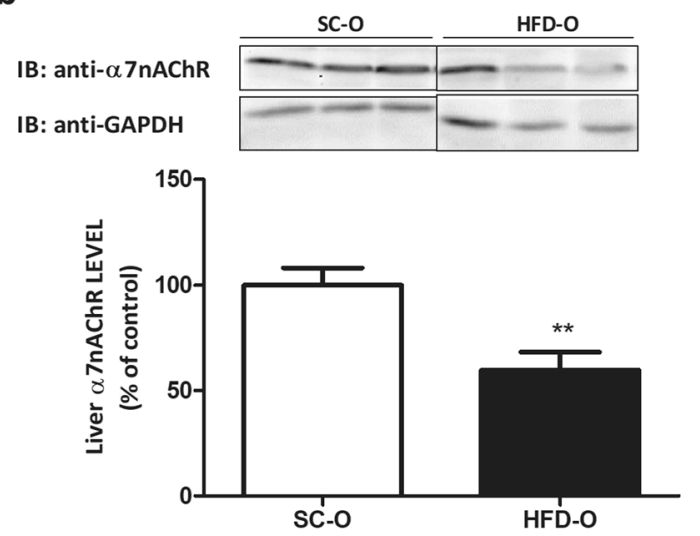

Figure 1. Nicotinic receptor expression in the offspring (P28). Hepatic $\alpha 7 \mathrm{nAChR}$ mRNA (a) and protein levels (b) were evaluated by Western blot in control and obese dams' offspring (P28). The percent expression of control (GAPDH) is shown (means $\pm \mathrm{SD}, \mathrm{n}=6$ pups per group). Black line of gel (Fig. $1 \mathrm{~b}$ ) indicates that gels/ blots were cropped from different parts of the same gel. Statistical significance was analysed by Student's t-test $(* \mathrm{p}<0.05, * * \mathrm{p}<0.01)$.

In previous studies, we showed that diet-induced maternal obesity leads to increased susceptibility to obesity and impairment of insulin signalling in offspring in early and late life ${ }^{9}$, inflammatory pathway activation ${ }^{10-12}$ and hypothalamic endoplasmic reticulum stress ${ }^{12}$. Recently, we also showed that high-fat diet (HFD) during pregnancy and lactation impairs the cholinergic anti-inflammatory pathway in liver and white adipose tissue and exacerbates cytokine production in response to LPS ${ }^{11}$. Therefore, it is possible that HFD could enable the expression and secretion of inflammatory cytokines and finally contribute to the development of insulin resistance in the offspring. Here, we evaluated the effect of maternal HFD consumption in the liver inflammatory response, cholinergic pathway and insulin AKT activation in the offspring recently weaned. To establish the correlation among liver $\alpha 7 \mathrm{nAChR}$ activation, inhibition of inflammatory pathways and improvement in the insulin signalling, we used mouse hepatoma cell line treated with saturated fatty acid (SFA) in the presence or absence of pharmacological agonists of $\alpha 7 \mathrm{nAChR}$.

\section{Results}

Maternal HFD consumption reduces liver $\alpha 7 n A C h R$ expression and activates inflammatory

pathway. First, we monitored the body weight gain of dams fed either standard chow (SC) or a high-fat diet (HFD). Body weight gain in the adaptation period was higher in HFD-fed than SC-fed dams (Fig. S1a). During pregnancy the body weight gain was similar for both groups (Fig. S1b) but during the suckling period (Fig. S1c) body weight gain was higher in offspring from HFD-fed dams (HFD-O) than in offspring from SC-fed dams (SC-O). Next, we evaluated the expression of $\alpha 7 \mathrm{nAChR}$ in liver of HFD-O and SC-O mice. The mRNA level of $\alpha 7 \mathrm{nAChR}$ was significantly higher in HFD-O than SC-O mice (2.5-fold). However, the amount of liver $\alpha 7 \mathrm{nAChR}$ protein was diminished (1.7-fold) in HFD-O compared to SC-O mice (Fig. 1a,b). Since $\alpha 7 \mathrm{n} A C h R$ has important role in the inhibition of inflammatory cytokines expression, we evaluated the hepatic IL-1 $\beta$ and TNF- $\alpha$ levels. Both cytokines presented higher levels in HFD-O than SC-O mice (Fig. 2a,b). On the other hand, JNK phosphorylation (pJNK) in liver was significantly reduced (2.1-fold), while IKK phosphorylation (pIKK) showed a tendency to increase in HFD-O compared to SC-O mice (Fig. 2c,d). LPS injection in SC-O and HFD-O mice increased hepatic TNF- $\alpha$ mRNA level in both groups, but the effect was more exacerbated in HFD-O compared to SC-O mice (14- and 1.4-fold, respectively) (Fig. 2e).

Maternal HFD consumption impairs AKT phosphorylation stimulated by insulin. AKT phosphorylation is classically affected by inflammatory pathways. To investigate insulin resistance development in the offspring of obese dams, we evaluated hepatic AKT phosphorylation stimulated by insulin using two protocols (in vivo and ex-vivo) (Fig. 3a,b). Both protocols showed that AKT phosphorylation stimulated by insulin was smaller in HFD-O than SC-O mice. Additionally, we evaluated insulin-stimulated phosphorylation of AKT in HFD-fed $\mathrm{KO} \alpha 7^{\mathrm{Alb}-\mathrm{Cre}}$ mice compared to HFD-fed WT mice. As can be observed in Fig. 3c, AKT phosphorylation was lower in $\mathrm{KO} \alpha 7^{\mathrm{Alb}-\mathrm{Cre}}$ mice than in WT mice.

PNU and nicotine reduce inflammatory pathway activation in hepatoma cell line induced by palmitate. We characterised first the inflammatory response to palmitate of Hepa-1c1c7 cell line. As shown in Fig. $4 \mathrm{a}$, cellular exposition to palmitate induced a slight increase in $\alpha 7 \mathrm{nAChR}$ expression, but neither PNU nor nicotine changed the expression of $\alpha 7 \mathrm{nAChR}$ significantly. To investigate the role of PNU in the activation of inflammatory pathways by the exposition to palmitate, we evaluated JNK phosphorylation (pJNK). As shown in the Fig. 4b, the exposition to palmitate increased (1.4-fold) pJNK level, but the addition of PNU reduced pJNK level significantly (76\%). The level of pIKK was also investigated, but treatment with palmitate did not alter the phosphorylation significantly (data not shown). Additionally, the treatment of cells with palmitate increased 
a

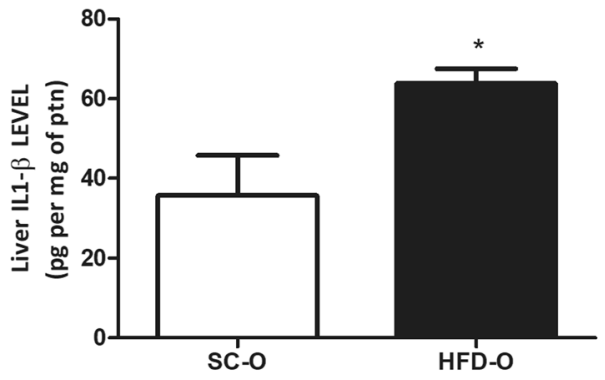

C

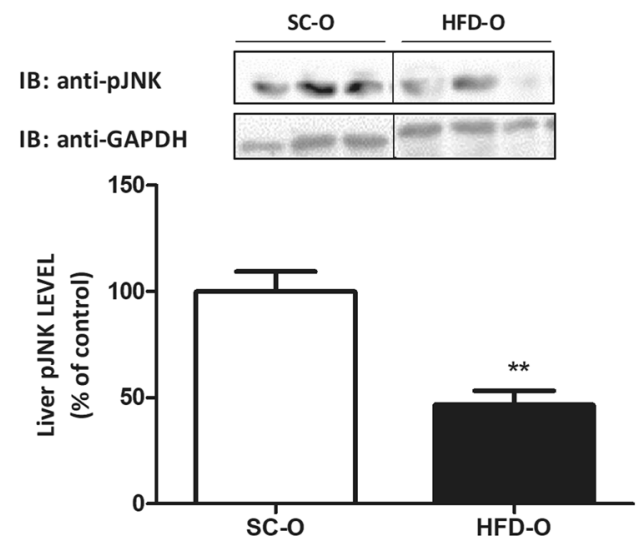

b

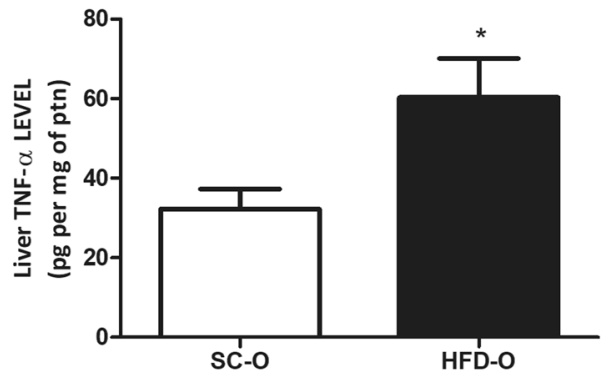

d

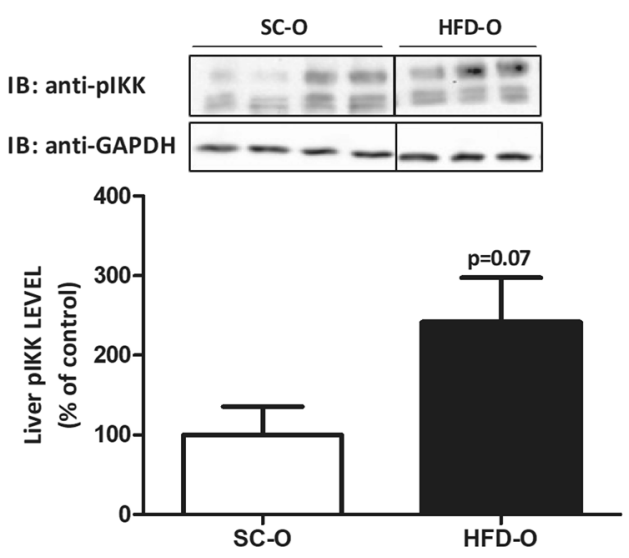

e

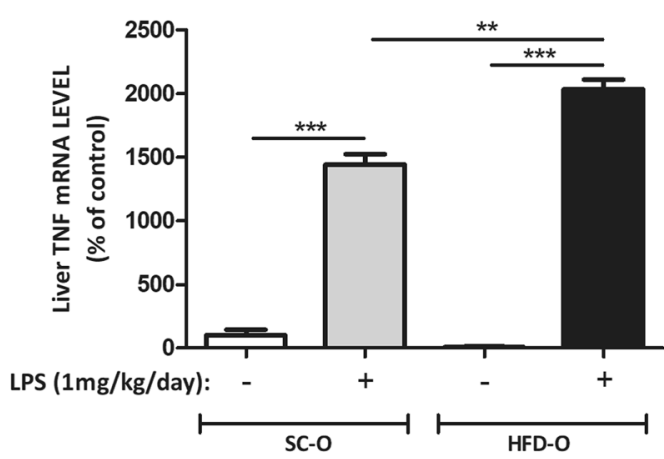

Figure 2. Cytokine expression in offspring's liver. Hepatic IL1- $\beta$ (a), TNF- $\alpha$ (b), pJNK (c) and pIKK (d) protein levels were evaluated by ELISA and Western blot in control and obese dams' offspring (P28). The expression of the cytokines is shown per mg of protein content. Hepatic TNF- $\alpha$ (e) mRNA levels were evaluated by RT-PCR in control and obese dams' offspring (P28) after treatment with LPS i.p. $1 \mathrm{mg}$ per kg body weight for 72 hours. The percent expression of control (GAPDH) is shown (means $\pm S D, n=5$ pups per group). Black line of gel (Fig. 2c,d) indicates that gels/blots were cropped from different parts of the same gel. Statistical significance was analysed by ANOVA and Bonferroni post-hoc tests $(* \mathrm{p}<0.05$, **p $<0.01$, ***p $<0.001$ ), or Student's t-test for analysis of two groups $(\mathrm{p}=0.07)$.

TNF- $\alpha$ mRNA (Fig. 4d) (3.1-fold) and showed a tendency $(\mathrm{p}=0.06)$ to increase the levels of IL-6 mRNA (Fig. 4c). PNU was efficient in reducing IL-6 mRNA level induced by palmitate (Fig. 4c), but to TNF- $\alpha$ mRNA levels, the effect was not significant. Similar results were observed in the presence of nicotine (Fig. 4c,d). AKT phosphorylation was used as a marker of the effect of inflammatory pathway on insulin signalling. As observed, insulin treatment of Hepa-1c1c7 cells increased (2.5-fold) AKT phosphorylation, but the previous treatment with palmitate reduced (52\%) the capacity of insulin to stimulate AKT phosphorylation (Fig. 4e) and increased JNK phosphorylation (Fig. 5b,d). The activation of $\alpha 7 \mathrm{nAChR}$ receptor prevented the harmful effect of palmitate on the insulin-stimulated AKT phosphorylation. As shown in Fig. 5a,c, insulin-stimulated AKT phosphorylation 
a
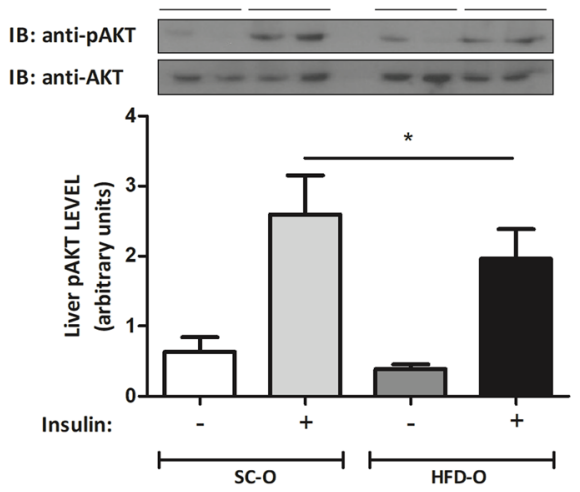

b

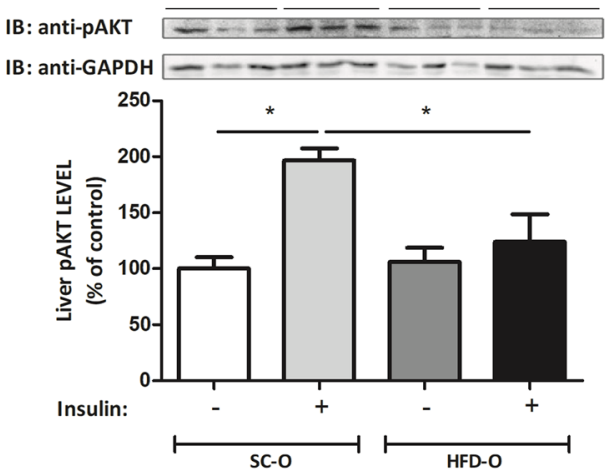

C

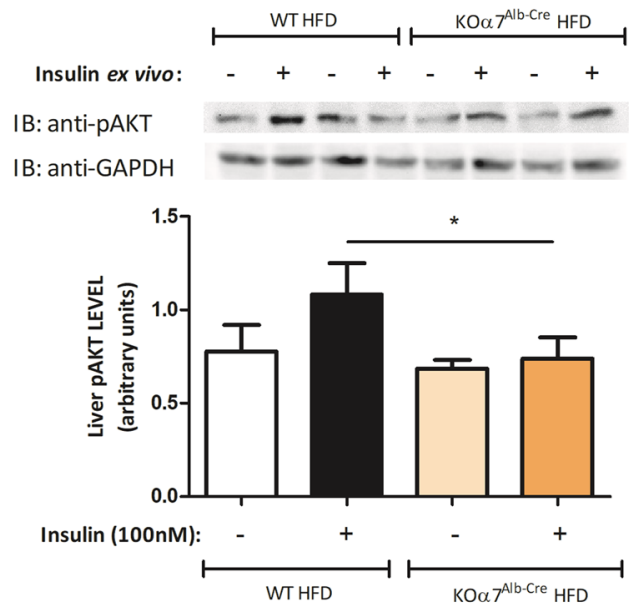

Figure 3. Impairment of the insulin signalling in the obese mother's offspring. Hepatic pAKT protein levels were evaluated by Western blot in control and obese dams' offspring (P28) after a bolus injection of saline or regular insulin (5 UI) through the abdominal cava vein. The expression of the protein was measured after 45 seconds of insulin administration. The percent expression of control (AKT) is shown (means $\pm S D, n=6$ per group). (a) Hepatic pAKT protein levels were evaluated by Western blot in control and obese dams' offspring (P28) after an ex vivo experiment where the tissue was treated with insulin $(100 \mathrm{nM})$ for 10 minutes in the cell media. The percent expression of control (GAPDH) is shown (means $\pm S D, n=6$ pups for $\mathrm{SC}-\mathrm{O}$ and $\mathrm{n}=4$ pups for HFD-O). (b) Hepatic pAKT protein levels were evaluated by Western blot in KO $\alpha 7^{\text {Alb-Cre }}$ and WT mice after an ex vivo experiment where the tissue was treated with insulin $(100 \mathrm{nM})$ for 10 minutes in the cell media. The percent expression of control (GAPDH) is shown (means $\pm S D, n=6$ pups for SC-O and $n=4$ pups for HFDO). (c) Statistical significance was analysed by Student's t-test for analysis of two groups $(* \mathrm{p}<0.05)$.

was increased (3.4-fold) while pJNK level was reduced (2.8-fold) in the presence of nicotine or PNU, agonists of $\alpha 7 \mathrm{nAChR}$ receptor (Fig. 5b,d).

\section{Discussion}

The activation of inflammatory pathways is known to induce insulin resistance in central and peripheral tissues $^{13-15}$. Saturated fatty acids and LPS can activate TLR4 receptor and stimulate inflammatory cytokines expression, leading to activation of serine kinases (JNK and IKK) that are responsible for inhibiting insulin signalling ${ }^{7,8,16}$. JNK and IKK activation are also observed in diet-induce obesity (DIO) and genetic models ${ }^{17,18}$. In metabolic programming, maternal obesity also contributes to the activation of inflammatory pathways, hypothalamic endoplasmic reticulum stress and damage to glucose homeostasis ${ }^{9-12}$.

Here, we showed that recently weaned offspring mice from dams fed with HFD during pregnancy and lactation have increased hepatic concentration of inflammatory cytokines (IL1 $\beta$ and TNF- $\alpha$ ) as well as phosphorylation of JNK and IKK. Moreover, liver TNF- $\alpha$ mRNA expression induced by LPS was more pronounced in HFD-O compared to SC-O mice. Interestingly, although $\alpha 7 \mathrm{nAChR}$ mRNA level was increased in HFD-O compared to SC-O mice, hepatic $\alpha 7 \mathrm{nAChR}$ protein level was reduced in HFD-O compared to SC-O mice. Since $\alpha 7 \mathrm{nAChR}$ gene expression was raised and protein level was diminished in HFD-O mice, post-translational modifications may be acting and stimulating $\alpha 7 \mathrm{nAChR}$ degradation. Nicotinic acetylcholine receptors are a target of the ubiquitin-proteasome system, as demonstrated in $\alpha 3, \alpha 7, \beta 2$ and $\beta 4$ subunits ${ }^{19,20}$, directing the ubiquitinated subunit for proteasomal degradation. HFD-O mice showed a slight increase in ubiquitination of $\alpha 7 \mathrm{nAChR}$ but 
a

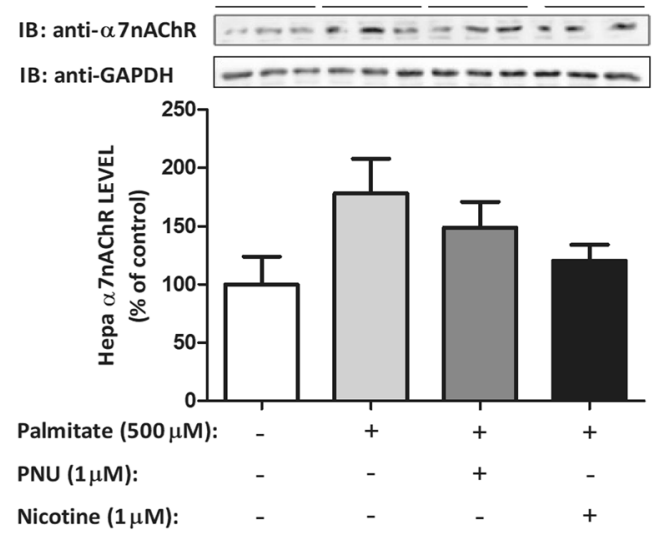

C

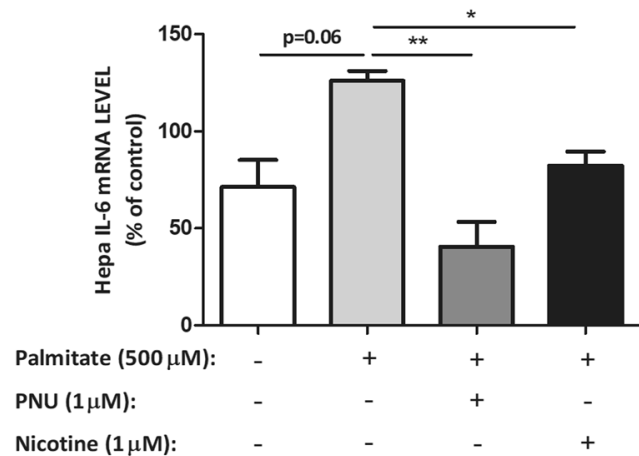

b
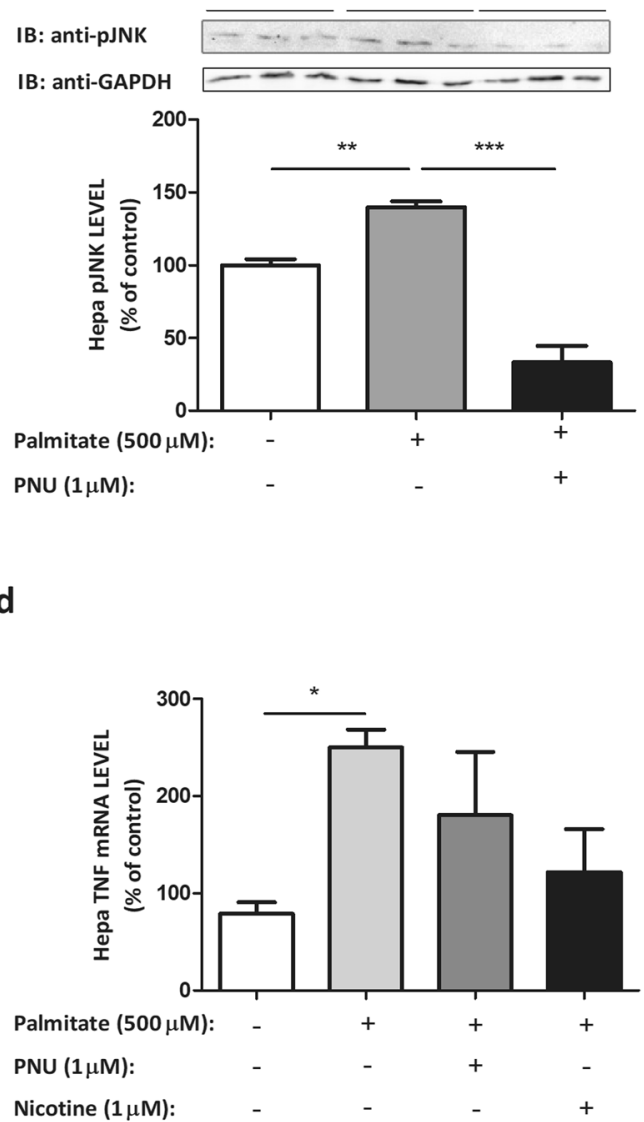

e

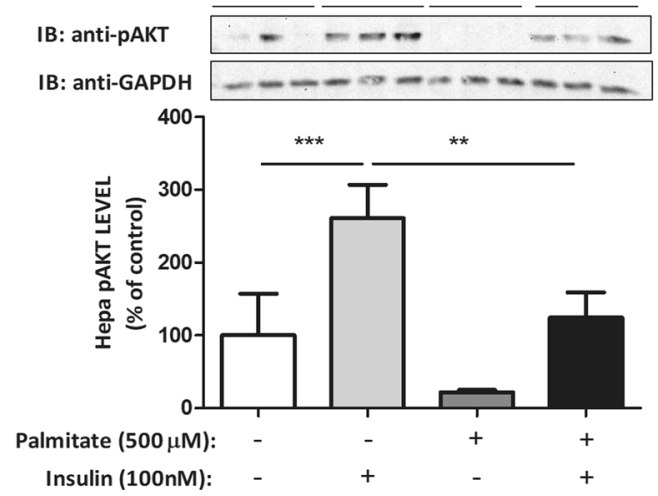

Figure 4. Palmitate and cholinergic agonists modulate the inflammatory pathway and insulin resistance in hepatocyte lineage. $\alpha 7 \mathrm{nAChR}(\mathbf{a})$, pJNK (b) evaluated by Western blot (WB), and IL-6 (c) and TNF- $\alpha$ (d) mRNA levels evaluated by RT-PCR, and pAKT (e) evaluated by WB in hepatoma cells lineage, Hepa-1clc7 $\left(\right.$ ATCC $^{\circledR}$ CRL-2026 $\left.{ }^{\mathrm{TM}}\right)$, after treatment with palmitate $(500 \mu \mathrm{M})$ for 3 hours and nicotine $(1 \mu \mathrm{M})$ or PNU $(1 \mu \mathrm{M})$ for 15 minutes, or insulin $(100 \mathrm{nM})$ for 10 minutes. The percent expression of control (GAPDH) is shown (means $\pm \mathrm{SD}, \mathrm{n}=3$ independent experiments with triplicate each). Statistical significance was analysed by ANOVA and Bonferroni post-hoc tests $(* \mathrm{p}<0.05, * * \mathrm{p}<0.01, * * * \mathrm{p}<0.001)$.

this was not significant. To further explore the role of the post-translational mechanism acting on the $\alpha 7 \mathrm{nAChR}$ protein level we evaluated the expression of RIC3, an important chaperone protein that influences the folding and assembly of $\alpha 7 \mathrm{nAChR}$ in the endoplasmic reticulum. However, RIC3 expression was no different in HFD-O compared to SC-O mice.

The receptor $\alpha 7 \mathrm{nAChR}$ is an important component of cholinergic anti-inflammatory pathway ${ }^{2,6}$. Although in a previous study, we also observed the negative effect of maternal HFD consumption on the hepatic $\alpha 7 \mathrm{nAChR}$ expression in the offspring, leading to higher susceptibility to activation of inflammatory pathway compared to 
a

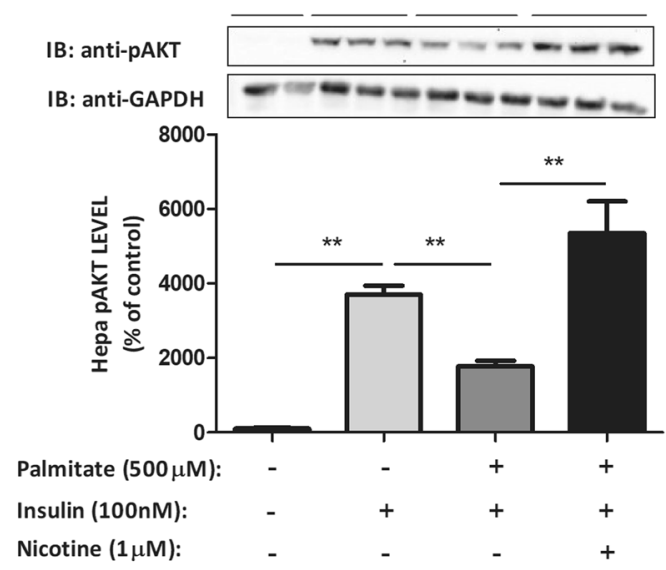

C

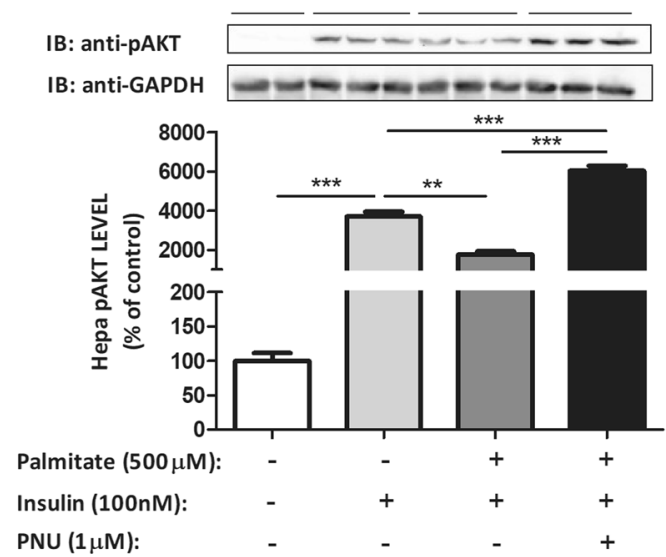

b
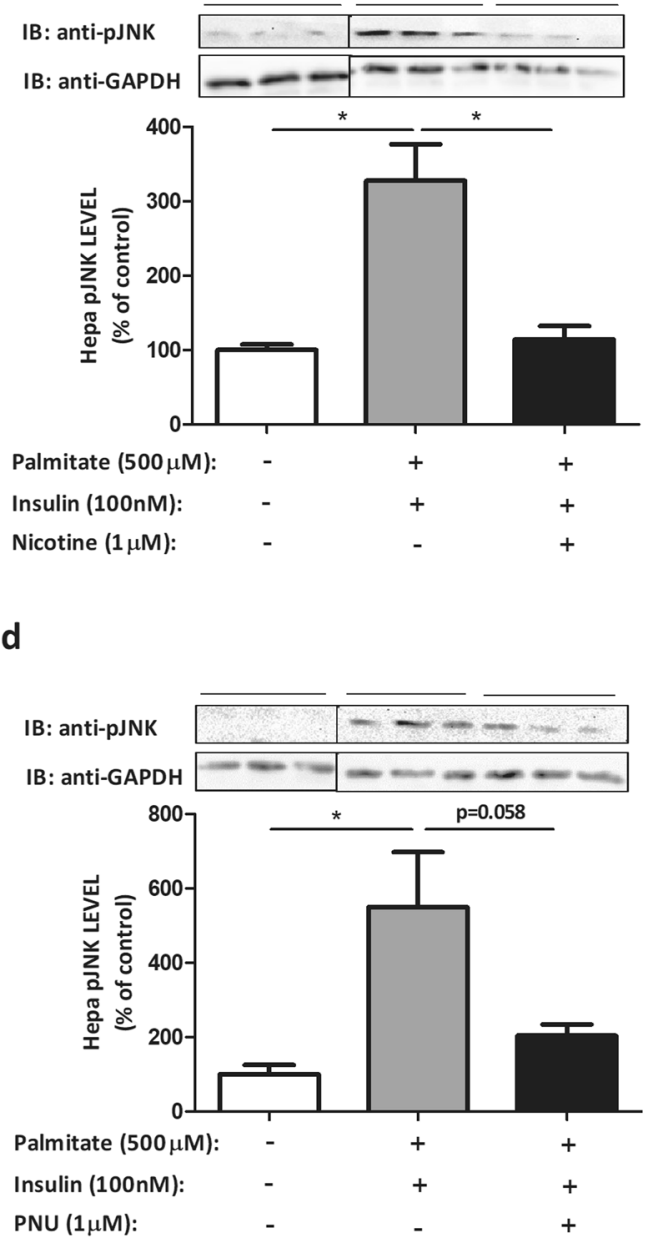

Figure 5. Cholinergic agonists improve the insulin signalling in hepatocyte lineage after treatment with palmitate. pAKT $(\mathbf{a}, \mathbf{c})$ and pJNK $(\mathbf{b}, \mathbf{d})$ protein levels were evaluated by Western blot in hepatoma cells lineage, Hepa-1c1c7 (ATCC ${ }^{\circledR}$ CRL-2026 ${ }^{\mathrm{TM}}$ ), after treatment with palmitate $(500 \mu \mathrm{M})$ for 3 hours, insulin $(100 \mathrm{nM})$ for 10 minutes and nicotine $(1 \mu \mathrm{M})$ or PNU $(1 \mu \mathrm{M})$ for 15 minutes. The percent expression of control (GAPDH) is shown (means $\pm S D, n=3$ independent experiments with triplicate each). Black line of gel (Fig. 5b,d) indicates that gels/blots were cropped from different parts of the same gel. Statistical significance was analysed by ANOVA and Bonferroni post-hoc tests, or Student's t-test for analysis of two groups $(* \mathrm{p}<0.05, * * \mathrm{p}<0.01$, $* * * \mathrm{p}<0.001)$.

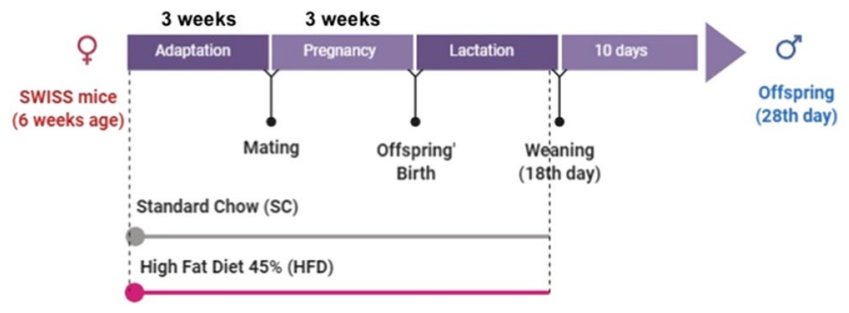

Figure 6. Experimental protocol of in vivo experiments.

SC-O mice ${ }^{11}$, we have not investigated the relationship with the development of insulin resistance. Here, HFD-O mice did not show difference in the basal glycaemia (data not shown), but AKT phosphorylation stimulated by insulin was reduced in HFD-O compared to SC-O mice. The present data, together with previous results showing that liver JNK phosphorylation and glucose production were increased in HFD-O, as indicated by pyruvate tolerance test (PTT) and liver PEPCK expression ${ }^{12}$, point to the development of insulin resistance. Although the focus of our investigation has been the liver, we showed in a previous study using the same model that white adipose 
tissue and soleus muscle also presented insulin resistance ${ }^{9}$. These effects were stable considering that insulin resistance in offspring from obese dams was detected until 82-days-old mice ${ }^{9}$.

Previously we showed that hepatic cyclic adenosine monophosphate (cAMP) response element binding protein (CREB) phosphorylation was reduced in HFD-O mice compared to SC-O mice ${ }^{11}$. CREB phosphorylation is induced by CAMP and it plays an important role in the recruitment of coactivators and activation of gluconeogenesis ${ }^{21-24}$. However, insulin signalling induces CREB-binding protein (CBP) phosphorylation, thus reducing the recruitment of coactivators and consequently gluconeogenesis ${ }^{25}$. Furthermore, CREB also participates in anti-inflammatory signalling induced by $\alpha 7 \mathrm{nAChR}$ activation. As demonstrated by Yoshikawa and colleagues,

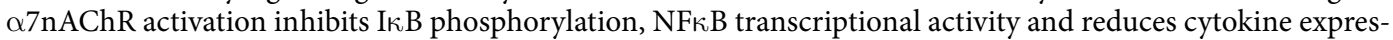
sion via $\mathrm{AKT}$ and $\mathrm{CREB}^{26}$. Additionally, Chiefari and colleagues, in a recent review, discussed the participation of high mobility group A1 (HMGA1) in glucose homeostasis ${ }^{27}$; these authors provided evidence of previous studies indicating that HMGA1, cAMP, protein kinase A (PKA) and CREB play an important role in gluconeogenesis activation. This pathway is inhibited by inactivation of HMGA1 due to insulin-induced HMGA1 phosphorylation $^{27}$. Considering the relation between $\alpha 7 \mathrm{nAChR}$ activation and CREB phosphorylation, HMGA1 could be an additional pathway modulated by the cholinergic receptor that has an important effect on glucose homeostasis. The development of insulin resistance in HFD-O mice may be related to diminished expression of $\alpha 7 \mathrm{nAChR}$ and reduction of the phosphorylated CREB level in hepatocytes. In this condition, inflammatory cytokine expression can damage insulin signalling and glucose homeostasis.

Recently, in an elegant study, Li et al. ${ }^{28}$ demonstrated that HFD consumption was more harmful to $\alpha 7 \mathrm{nAChR}^{-1-}$ than wild type (WT) mice. The HFD-fed $\alpha 7 \mathrm{nAChR}^{-1-}$ mice showed more pronounced hepatic lipid accumulation, macrophage infiltration and mRNA levels of TNF- $\alpha$, IL- 6 and IL- $1 \beta$ than HFD-fed WT mice. In addition, liver insulin signalling was significantly damaged in HFD-fed $\alpha 7 \mathrm{nAChR}^{-1-}$ mice compared to HFD-fed WT mice. Here, we showed that HFD-O mice has reduced expression of $\alpha 7 \mathrm{nAChR}$, which could predispose the mice to metabolic damages. However, differently of offspring from obese dams (HFD-O) explored in this manuscript, $\mathrm{Li}$ and colleagues ${ }^{28}$ demonstrated that HFD-fed WT mice presented increased liver expression of $\alpha 7 \mathrm{nAChR}$ over the period fed with HFD (8 weeks), implicating $\alpha 7 \mathrm{nAChR}$ in the development of non-alcoholic fatty liver disease (NAFLD). However, the authors showed that the specific $\alpha 7 \mathrm{nAChR}$ activation with PNU partly rescued the NAFLD phenotypes. Thus, maternal consumption of HFD can impair liver $\alpha 7 n A C h R$ expression and contribute to the early onset of inflammatory changes and homeostasis damage in the offspring. To further explore the participation of liver $\alpha 7 \mathrm{nAChR}$ in the development of insulin resistance, we investigated insulin-stimulated phosphorylation of AKT in HFD-fed KO $\alpha 7^{\mathrm{Alb}-\mathrm{Cre}}$ mice with deletion of $\alpha 7 \mathrm{nAChR}$ in the hepatocytes. AKT phosphorylation was lower in $\mathrm{KO} \alpha 7^{\mathrm{Alb}-\mathrm{Cre}}$ than WT mice, suggesting a protective effect of $\alpha 7 \mathrm{nAChR}$ in hepatocytes under inflammatory conditions.

Cholinergic anti-inflammatory pathway and $\alpha 7 \mathrm{nAChR}$ activation have been associated to attenuate inflammatory response in endotoxemia ${ }^{5}$, macrophage TNF- $\alpha$ release ${ }^{6}$, hepatic lipid accumulation and damage to glucose homeostasis ${ }^{28}$. However, the effects that were associated to the activation of cholinergic receptor could have come from different cell types present in the liver. Li et al. ${ }^{28}$ used knockout mice $\left(\alpha 7 \mathrm{nAChR}{ }^{-1-}\right)$ to evaluate the effect of HFD consumption on liver metabolic disturbances related to lipid accumulation. Although the findings are very important to relate $\alpha 7 \mathrm{nAChR}$ to the progression of NAFLD and insulin resistance, the effects described may have arisen from $\alpha 7 \mathrm{nAChR}$ located in hepatocytes or macrophages for example.

The controversy about inflammation triggered from interaction between saturated fatty acid and TLR $4^{29}$ has received more information recently ${ }^{30}$. However, cells incubated with saturated fatty acids showed increased inflammatory markers ${ }^{31,32}$. Thus, the exposition of cell culture to saturated fatty acid is an excellent model to stimulate inflammatory pathway activation and induce insulin resistance. The hepatoma cell line Hepa-1c1c7 $\left(\right.$ ATCC $^{\circledR}$ CRL-2026 ${ }^{\mathrm{TM}}$ ) exposed to palmitate presented increased JNK phosphorylation, IL- 6 and TNF- $\alpha$ mRNA compared to cells exposed to vehicle solvent alone. Moreover, these effects were accompanied by insulin resistance, as measured by reduced AKT phosphorylation stimulated by insulin in cells previously incubated with saturated fatty acid. To evaluate if $\alpha 7 \mathrm{nAChR}$ activation could reduce the inflammation and insulin resistance, we used nicotine and PNU, which are agonist of cholinergic receptor. Both agonists were efficient to reduce the inflammatory marker levels in cells exposed to saturated fatty acid and improve insulin signalling.

In conclusion, we demonstrated that $\alpha 7 \mathrm{nAChR}$ activation in hepatocytes is able to improve insulin signalling through inhibition of cytokines expression and JNK activation independent of $\alpha 7 \mathrm{nAChR}$ activation in macrophages. Moreover, although HFD consumption increased liver expression of $\alpha 7 \mathrm{nAChR}$, as demonstrated by $\mathrm{Li}$ and colleagues ${ }^{28}$, here we showed that maternal HFD consumption diminishes hepatic $\alpha 7 \mathrm{nAChR}$ expression, increases hepatic cytokines mRNA level and induces insulin resistance in the offspring.

\section{Methods}

Animals. Swiss female mice aged 5-6 weeks were obtained from the Multidisciplinary Center for Biological Research at University of Campinas (Campinas, Brazil). The mice were held in a temperature-controlled environment ( $12 \mathrm{~h}$ light/dark cycle). Ethics approval was obtained from the State University of Campinas Ethics Committee (Protocol 4328-1) and all experiments were performed in accordance with relevant guidelines and regulations for the care and use of laboratory animals. Dams were randomly separated in two different groups ( $\mathrm{n}=10$ dams per group), fed with either high-fat diet (HFD) or standard chow diet (SC) (NUVILAB ${ }^{\circledR}$ Cr-1, Nuvital, PR-Brazil) (Table 1), ad libitum during pregnancy and lactation. After birth the litter size was adjusted to eight animals per litter. HFD was prepared in our laboratory according to the AIN-93G but modified for highfat content $(45 \%)$ as previously described ${ }^{12}$. Male offspring were weaned on the $18^{\text {th }}$ day after birth and fed with standard chow until $28^{\text {th }}$ day (Fig. 6). Mice with liver-specific Chrna7 deficiency were generated using the CreloxP system. To obtain KO $\alpha 7^{\text {Alb-Cre }}$ mice we mated Chrna $7^{\text {flox/flox }}\left(\mathrm{B} 6(\mathrm{Cg})-\mathrm{Chrna} 7^{\text {tm1.1Ehs }} /\right.$ YakelJ) and Alb-Cre (B6. 


\begin{tabular}{|c|c|c|}
\hline Ingredients & $\begin{array}{l}\text { Standard Chow } \\
(\mathrm{g} \%)\end{array}$ & $\begin{array}{l}\text { High Fat Diet } \\
(\mathrm{g} \%)\end{array}$ \\
\hline Carbohydrates $^{(\mathrm{a})}$ & 55.0 & 41.2 \\
\hline Net Protein ${ }^{(b)}$ & 22.5 & 20.8 \\
\hline Fat content $^{(c)}$ & 4.5 & 23.6 \\
\hline Fibrous matter & 8.0 & 5.8 \\
\hline Ash matter & 10.0 & 8.6 \\
\hline Total & \begin{tabular}{|l|l|}
100.0 \\
\end{tabular} & 100.0 \\
\hline
\end{tabular}

Table 1. Nutritional composition of experimental and standard chow diet. Standard Chow: NUVILAB ${ }^{\circledR}$ Cr-1, Nuvital, PR-Brazil. (a)Starch and saccharose. (b)Vegetal protein - from wheat and corn (added lysine and methionine). (c)Soy oil and lard.

Cg-Speer6-ps1 ${ }^{\mathrm{Tg}(\mathrm{Alb}-\mathrm{cre}) 21 \mathrm{Mgn} / \mathrm{J})}$ mice. Both lineages were purchased from The Jackson Laboratory. Chrna7 $7^{\text {flox/flox }}$ animals were used for the control (WT). The male mice were maintained on a 45\% HFD for 8 weeks.

Anaesthesia and tissue extraction. Mice were anaesthetised with a mixture containing ketamine (139.2 $\left.\mathrm{mg} \mathrm{kg}^{-1} \mathrm{bw}\right)$, diazepam $\left(4 \mathrm{mg} \mathrm{kg}^{-1} \mathrm{bw}\right)$ and xylazine $\left(18.4 \mathrm{mg} \mathrm{kg}^{-1} \mathrm{bw}\right)$ and subsequently euthanized by decapitation for the tissue extraction. Tissue samples were frozen in liquid nitrogen and stored at $-80^{\circ} \mathrm{C}$ until processing. When necessary, the samples were plated on a culture plate containing cold Dulbecco's modified Eagle medium (DMEM; Invitrogen, USA) for analysis of the insulin signalling pathway.

Cytokine level measurements. Mice offspring were sacrificed on the $28^{\text {th }}$ day after an overnight fasting period. Liver samples were collected and homogenised in extraction buffer with phosphate-buffered saline (PBS) and protease inhibitor. Afterwards, the samples were centrifuged for $30 \mathrm{~min}$ at $25^{\circ} \mathrm{C}$ for the protein extraction. The IL- $1 \beta$ and TNF- $\alpha$ cytokine levels were measured using an Elisa Duo Set Kit (R\&D System, Minneapolis, MN, USA).

Western blot analysis. Tissues were homogenised in freshly prepared ice-cold buffer (1\% v/v Triton $\mathrm{X}-100,0.1 \mathrm{~mol} / \mathrm{L}$ Tris, $\mathrm{pH} 7.4,0.1 \mathrm{~mol} / \mathrm{L}$ sodium pyrophosphate, $0.1 \mathrm{~mol} / \mathrm{L}$ sodium fluoride, $0.01 \mathrm{~mol} / \mathrm{L}$ EDTA, $0.01 \mathrm{~mol} / \mathrm{L}$ sodium vanadate, $0.002 \mathrm{~mol} / \mathrm{L}$ PMSF and $0.01 \mathrm{mg} / \mathrm{mL}$ aprotinin). The supernatant protein was separated by centrifugation $(10,000 \times \mathrm{g})$ for $30 \mathrm{~min}$ at $4{ }^{\circ} \mathrm{C}$, and the protein concentration was determined using Biuret dye-bleeding method. Samples were resuspended in Laemmli sample buffer and boiled for 5 min before separation by SDS-PAGE using a miniature slab gel apparatus (BioRad, Richmond, CA, USA). Electrotransfer of proteins from the gel to a nitrocellulose membrane was performed for $10 \mathrm{~min}$ in a transfer buffer that contained methanol and SDS. These membranes were incubated overnight at $4{ }^{\circ} \mathrm{C}$ with specific antibodies: $\alpha 7 \mathrm{nAChR}$ (bs-1049R; Bioss Antibodies ${ }^{\odot}$ ), Phospo-JNK (\#9255; Cell Signaling ${ }^{\odot}$ ), Phospo-AKT (\#4060) and Phospo-IKK (\#2697). Then, after washout with a Tris-buffered saline (TBS)-Tween 20 (TTBS; $10 \mathrm{mmol} / \mathrm{L}$ Tris, $150 \mathrm{mmol} / \mathrm{L}$ $\mathrm{NaCl}, 0.5 \%$ Tween 20 ), the nitrocellulose membranes were probed with goat peroxidase-conjugated secondary antibodies (KPL, Gaithersburg, MD, USA) for 2 hours in room temperature. Proteins were detected by chemiluminescence kit (SuperSignal West Pico Chemiluminescent Substrate, Thermo Fisher Scientific, MA, USA) and bands were evaluated by densitometry using Scion Image software (ScionCorp, MD, USA). The intensities of the bands were normalised to those of total protein or loading control (GAPDH or $\beta$-actin).

RT-PCR analysis. Hepatic frozen tissue was homogenised in TRIzol reagent (Life Technologies Corporation, CA, USA) for RNA extraction. After $5 \mathrm{~min}$ at room temperature, the chloroform was added to the homogenate. Following centrifugation, the RNA phase was precipitated with isopropyl alcohol and then washed with 75 and $100 \%$ ethyl alcohol. After drying, the pellet was resuspended in ultra-pure water stored at $-80^{\circ} \mathrm{C}$. RNA was quantified in NanoDrop ND-2000 (Thermo Electron, USA). Reverse transcription was performed with $3 \mu \mathrm{g}$ of total RNA using High-Capacity cDNA Reverse Transcription kit (Life Technologies Corporation). Relative expression was determined using the Taqman detection system and primers for the following target genes: CHRNA7 (Mm01312230_m1; Thermo Fisher Scientific, USA), IL6 (146868493; Integrated DNA Technologies-IDT, USA), TNFo (Mm00443258_m1; Thermo Fisher Scientific, USA), IL1 $\beta$ (Mm00434228_m1; Thermo Fisher Scientific, USA). GAPDH (4351309; Applied Biosystems, USA) was used as endogenous control. Real time PCR was performed on an AB/Prism 7500 fast platform. Data were analysed using the sequence detection system 2.0.5.

AKT phosphorylation stimulated by insulin. Ex vivo analysis. Male mice offspring were euthanized to collect liver tissue samples as described previously. Fragments of hepatic tissue were incubated at $37^{\circ} \mathrm{C}$ for 2-4 hours in 24-well culture plates containing DMEM low glucose (Invitrogen, USA). Next, the medium was removed, and the tissue washed with $1 \mathrm{X}$ PBS. Tissue fragments were incubated at $37^{\circ} \mathrm{C}$ for 10 minutes in 24 -well culture plates containing $100 \mathrm{nM}$ insulin in DMEM low glucose (Invitrogen, USA). Tissues were then collected, froze in liquid nitrogen and stored at $-80^{\circ} \mathrm{C}$ until processing for Western blot analysis.

In vivo analysis. To evaluate AKT phosphorylation in vivo, a bolus injection of saline or 5 UI regular insulin (Humulin, Eli Lilly and Company, USA) was administered through the abdominal cava vein. Fragments of liver were extracted 45 seconds after insulin/saline injection. For animals stimulated with insulin, the delta value (value after stimulation - value before stimulation) was considered for the statistical analysis. Tissue samples were frozen in liquid nitrogen and stored at $-80^{\circ} \mathrm{C}$ until processing. 
LPS treatment. To induce inflammatory response, mice were treated with LPS diluted in sterile saline and administered intraperitoneally (IP) once a day for three days $\left(1 \mathrm{mg} \mathrm{kg}^{-1} \mathrm{bw}\right.$ - IP). Mice were euthanized 2 hours after LPS treatment, and fragments of liver were collected, froze in liquid nitrogen and stored at $-80^{\circ} \mathrm{C}$ until processing.

In vitro experiments. Hepatoma cell line, Hepa-1c1c7 (ATCC ${ }^{\circledR}$ CRL-2026 ${ }^{\mathrm{TM}}$ ), derived from mice was used to evaluate the ability of the cholinergic pathway to modulate AKT phosphorylation induced by insulin. Cells were cultivated in alpha modified Eagle's medium ( $\alpha$ MEM; Invitrogen, USA) supplemented with 10\% foetal bovine serum (Invitrogen, USA) and $1 \%$ penicillin $(100 \mathrm{U} / \mathrm{mL}) /$ streptomycin $(100 \mu \mathrm{g} / \mathrm{mL})$ (Invitrogen, USA) at $37^{\circ} \mathrm{C}$ and $5 \% \mathrm{CO}_{2}$.

Cells were treated with $500 \mu \mathrm{M}$ palmitate (palmitic acid from Sigma-Aldrich at $500 \mu \mathrm{M}$ was first diluted in $\mathrm{NaOH}$ conjugated to BSA (3:1) for 45 minutes at $37^{\circ} \mathrm{C}$ ) for 3 hours in 6-well culture plate. The protein content was extracted and analysed by Western blotting. When necessary, $1 \mu$ M PNU-282987 (P6499-10MG; Sigma-Aldrich, Brazil) or $1 \mu \mathrm{M}$ nicotine (N0267-100MG; Sigma-Aldrich, Brazil) was added to the medium for 15 minutes after palmitate treatment. To evaluate the insulin signalling, cells were treated with $100 \mathrm{nM}$ insulin (Humulin, Eli Lilly and Company, USA) for 10 minutes after the 3 hours of palmitate treatment.

Data presentation and statistical analysis. All results are presented as the mean $\pm \mathrm{SD}$. Student's $t$-test of unpaired samples and analysis of variance (ANOVA) for multiple comparisons were carried out after confirmation of normal distribution using the Kolmogorov-Smirnov test. ANOVA was followed by the Bonferroni post-hoc test and used when differences among more than two groups were analysed. Statistical significance for all analyses was set at $p<0.05$. All statistical comparisons were performed using GraphPad Prism 6.01 software (http://www.graphpad.com/scientific-software/prism/).

Received: 1 May 2019; Accepted: 10 December 2019;

Published online: 08 January 2020

\section{References}

1. Pavlov, V. A. \& Tracey, K. J. The vagus nerve and the inflammatory reflex-linking immunity and metabolism. Nat. Rev. Endocrinol. 8, 743-54 (2012)

2. Tracey, K. J. The inflammatory reflex. Nature 420, 853-859 (2002).

3. Rosas-Ballina, M. \& Tracey, K. J. The Neurology of the Immune System: Neural Reflexes Regulate Immunity. Neuron 64, 28-32 (2009).

4. Cheng, P. Y., Lee, Y. M., Law, K. K., Lin, C. W. \& Yen, M. H. The involvement of AMP-activated protein kinases in the antiinflammatory effect of nicotine in vivo and in vitro. Biochem. Pharmacol. 74, 1758-1765 (2007).

5. Pavlov, V. A. et al. Selective $\alpha 7$-nicotinic acetylcholine receptor agonist GTS-21 improves survival in murine endotoxemia and severe sepsis. Crit. Care Med. 35, 1139-1144 (2007).

6. Wang, H. et al. Nicotinic acetylcholine receptor alpha7 subunit is an essential regulator of inflammation. Nature 421, 384-388 (2003).

7. McNelis, J. C. \& Olefsky, J. M. Macrophages, Immunity, and Metabolic Disease. Immunity 41, 36-48 (2014).

8. Lackey, D. E. \& Olefsky, J. M. Regulation of metabolism by the innate immune system. Nat. Rev. Endocrinol. 12, 15-20 (2016).

9. De Fante, T. et al. Diet-induced maternal obesity alters insulin signalling in male mice offspring rechallenged with a high-fat diet in adulthood. PLoS ONE 11, 1-22 (2016).

10. Ashino, N. G. et al. Maternal high-fat feeding through pregnancy and lactation predisposes mouse offspring to molecular insulin resistance and fatty liver. J. Nutr. Biochem. 23, 341-348 (2012).

11. Payolla, T. B. et al. High-fat diet during pregnancy and lactation impairs the cholinergic anti-inflammatory pathway in the liver and white adipose tissue of mouse offspring. Mol. Cell. Endocrinol. 422, 192-202 (2016).

12. Melo, A. M. et al. Hypothalamic endoplasmic reticulum stress and insulin resistance in offspring of mice dams fed high-fat diet during pregnancy and lactation. Metabolism. 63, 682-692 (2014).

13. De Souza, C. T. et al. Consumption of a fat-rich diet activates a proinflammatory response and induces insulin resistance in the hypothalamus. Endocrinology 146, 4192-4199 (2005).

14. Milanski, M. et al. Saturated Fatty Acids Produce an Inflammatory Response Predominantly through the Activation of TLR4 Signaling in Hypothalamus: Implications for the Pathogenesis of Obesity. J. Neurosci. 29, 359-370 (2009).

15. Chen, W., Balland, E. \& Cowley, M. A. Hypothalamic Insulin Resistance in Obesity: Effects on Glucose Homeostasis. Neuroendocrinology 104, 364-381 (2017).

16. Jia, L. et al. Hepatocyte Toll-like receptor 4 regulates obesity-induced inflammation and insulin resistance. Nat. Commun. 5, 3878 (2014).

17. Jais, A., Brüning, J. C., Jais, A. \& Brüning, J. C. Hypothalamic inflammation in obesity and metabolic disease Find the latest version: Hypothalamic inflammation in obesity and metabolic disease. J Clin Invest. 127, 24-32 (2017).

18. Park, J. H., Yoo, Y., Han, J. \& Park, Y. J. Altered expression of inflammation-associated genes in the hypothalamus of obesity mouse models. Nutr. Res., https://doi.org/10.1016/j.nutres.2018.06.006 (2018).

19. Rezvani, K., Teng, Y. \& De Biasi, M. The ubiquitin-proteasome system regulates the stability of neuronal nicotinic acetylcholine receptors. J. Mol. Neurosci. 40, 177-84 (2010).

20. Rezvani, K., Teng, Y., Shim, D. \& De Biasi, M. Nicotine Regulates Multiple Synaptic Proteins by Inhibiting Proteasomal Activity. J. Neurosci. 27, 10508-10519 (2007)

21. Herzig, S. et al. CREB regulates hepatic gluconeogenesis through the coactivator PGC-1. Nature 413, 179-183 (2001).

22. Chrivia, J. C. et al. Phosphorylated CREB binds specifically to the nuclear protein CBP. Nature 365, 855-859 (1993).

23. Kwok, R. P. S. et al. Nuclear protein CBP is a coactivator for the transcription factor CREB. Nature 370, 223-226 (1994)

24. Altarejos, J. Y. \& Montminy, M. CREB and the CRTC co-activators: sensors for hormonal and metabolic signals. Nat. Rev. Mol. Cell Biol. 12, 141-51 (2011).

25. Zhou, X. Y. et al. Insulin regulation of hepatic gluconeogenesis through phosphorylation of CREB-binding protein. Nat. Med. 10, 633-637 (2004).

26. Yoshikawa, H. et al. Nicotine inhibits the production of proinflammatory mediators in human monocytes by suppression of I- $\kappa \mathrm{B}$ phosphorylation and nuclear factor- $\kappa \mathrm{B}$ transcriptional activity through nicotinic acetylcholine receptor $\alpha 7$. Clin. Exp. Immunol. 146, 116-123 (2006).

27. Chiefari, E. et al. Transcriptional regulation of glucose metabolism: The emerging role of the HMGA1 chromatin factor. Front. Endocrinol. (Lausanne). 9, 1-14 (2018). 
28. Li, D. J. et al. Nicotinic acetylcholine receptor $\alpha 7$ subunit improves energy homeostasis and inhibits inflammation in nonalcoholic fatty liver disease. Metabolism. 79, 52-63 (2018).

29. Shi, H. et al. TLR4 links innate immunity and fatty acid - induced insulin resistance Find the latest version: TLR4 links innate immunity and fatty acid - induced insulin resistance. J. Clin. Invest. 116, 3015-3025 (2006).

30. Lancaster, G. I. et al. Evidence that TLR4 Is Not a Receptor for Saturated Fatty Acids but Mediates Lipid-Induced Inflammation by Reprogramming Macrophage Metabolism. Cell Metab. 27, 1096-1110.e5 (2018).

31. Kim, S. M., McIlwraith, E. K., Chalmers, J. A. \& Belsham, D. D. Palmitate induces an anti-inflammatory response in immortalized microglial BV-2 and IMG cell lines that decreases TNF $\alpha$ Levels in mHypoE-46 hypothalamic neurons in co-culture. Neuroendocrinology 107, 387-399 (2019).

32. Yang, J. et al. Quercetin protects obesity-induced hypothalamic inflammation by reducing microglia-mediated inflammatory responses via HO-1 induction. Nutrients 9, 1-14 (2017).

\section{Acknowledgements}

This work was supported by grants from the Coordination for the Improvement of Higher Education Personnel (CAPES), São Paulo Research Foundation - FAPESP (Grant Number 2016/23484-1 and 13/07607-8) and CNPq (Grant Number 409779/2018-0). The authors AST, MM, and MAT belong to the Obesity and Comorbidities Research Center - Sao Paulo Research Foundation. National Council for Scientific and Technological Development (CNPq) and São Paulo Research Foundation - FAPESP (grant \#16/23484-1 and \#13/07607-8). The funders had no role in study design, data collection or analysis, decision to publish and preparation of the manuscript.

\section{Author contributions}

S.O.C., C.M.S., P.G.L. and J.O.S. performed most of the experiments and to data analysis. T.C. and H.G.R. performed ELISA experiment. S.O.C., A.S.T. and M.A.T. writing original draft. L.M.I.S. and M.M. provide assistance to article supervision and discussion of results. M.M., A.S.T., L.M.I.S. and M.A.T. funding acquisition. All the authors approved the final manuscript.

\section{Competing interests}

The authors declare no competing interests.

\section{Additional information}

Supplementary information is available for this paper at https://doi.org/10.1038/s41598-019-56880-3.

Correspondence and requests for materials should be addressed to M.A.T.

Reprints and permissions information is available at www.nature.com/reprints.

Publisher's note Springer Nature remains neutral with regard to jurisdictional claims in published maps and institutional affiliations.

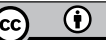

Open Access This article is licensed under a Creative Commons Attribution 4.0 International License, which permits use, sharing, adaptation, distribution and reproduction in any medium or format, as long as you give appropriate credit to the original author(s) and the source, provide a link to the Creative Commons license, and indicate if changes were made. The images or other third party material in this article are included in the article's Creative Commons license, unless indicated otherwise in a credit line to the material. If material is not included in the article's Creative Commons license and your intended use is not permitted by statutory regulation or exceeds the permitted use, you will need to obtain permission directly from the copyright holder. To view a copy of this license, visit http://creativecommons.org/licenses/by/4.0/.

(c) The Author(s) 2020 